\title{
NUTRIC̣ÃO PARENTERAL: PROGRAMA DE ASSISTÊNCIA DOMICILIÁRIA
}

\author{
Cynthia Maria da Silva* \\ Isabel Cristina Kowal Olm** \\ Rúbia Aparecida Lacerda***
}

\begin{abstract}
RESUMO - A Nutrição Parenteral Prolongada (NPP) tem sido objeto de estudos avançados e de resultados comprovadamente satisfatórios em pacientes com diversas patologias. Tentativas com sucesso têm sido levadas a efeito, direcionando o paciente com NPP de volta ao seu domicílio. Com base nisto, as autoras propõem a criação nos Hospitais de Ensino, de Programas de Assistência Domiciliária para estes pacientes. Apresentam neste trabalho um modelo para operacionalização deste Programa, baseado na atuação da Equipe Multiprofissional. É dado ênfase ao trabalho da enfermeira, dirigido para a assistência e educação do paciente bem como a de seus familiares, visando capacitá-los para o auto-cuidado.
\end{abstract}

\begin{abstract}
The Total Parenteral Nutrition (TPN) has been the purpose of advanced studies with results having been adequately corroborated among patients in need of nutritional support. A great number of endeavours have been made to allow the TPN patient to return home, and based on the findings in this study the authors suggest the development of Training Hospitals and Home Assistence Programs in their behalf. The pattern here presented gives evidence of the possibilities a multiprofessional support team has in performing this Program. The nurse's work as well as her responsabilities have been emphasized, aimed as they are at the assistance and education of patients and their kins in order to render them capable of self care.
\end{abstract}

\section{INTRODUC̣ÃO}

\section{Conceito de Nutrição Parenteral (N.P.)}

O objetivo da nutrição parenteral é assegurar um aporte hidro-eletrolítico e calórico-protéico cotidiano, suficiente para manutenção do paciente por tanto tempo quanto seja necessário (SOLASSOL \& JOYEUX, 1980).

Somente neste século é que houve o desenvolvimento tecnológico suficiente para que permitisse o preparo de soluções passíveis de serem administradas na veia e que contivessem os elementos nutritivos da dieta alimentar por via oral, sendo eles: proteínas, lipídios, carbohidratos, vitaminas, água e sais minerais. Essas soluções são formadas pelas unidades elementares destes elementos (soluções de aminoácidos em forma cris- talina, soluções concentradas de carbohidratos, emulsões gordurosas à base de óleo de soja e soluções de vitaminas hidro e lipossolúveis, bem como os sais minerais e oligoelementos necessários). (DICKSTEIN et alii, 1979)

\section{Tipos de Nutrição Parenteral}

Atualmente, utilizam-se três modalidades de nutrição parenteral:

a. Hiperalimentação Parenteral - é a mais conhecida e talvez a mais importante sendo originalmente introduzida em 1968 por DUDRICK et alii (1979). Fornecese ao paciente uma solução hipertônica, hiperosmolar, constituída basicamente de glicose à $50 \%$, solução de aminoácidos, em geral a 10\%, todas as vitaminas, uma mistura de eletrólitos que devem incluir somente fon-

\footnotetext{
* Enfermeira Mestranda da EEUSP. 10 Tenente do Quadro Feminino de Oficiais da Aeronáutica e Chefe da Subdivisão Enfermagem do Hospital Aeronáutico de S. Paulo.

** Enfermeira Mestranda da EEUSP. Prof. Aux. Depto. de Enfermagem da Escola Paulista de Medicina.

${ }^{* * *}$ Enfermeira. Mestranda da EEUSP. Prof. Aux. Escola de Enfermagem da Univ. Campinas (IJNICAMP).
} 
tes de sódio, potássio, magnésio, cálcio e fosfato e, quando possível, oligoelementos. Esta mistura deve ser obrigatoriamente infundida através de cateter na veia cava superior.

b. Nutrição Parenteral Total - cronologicamente mais antiga que a anterior, porém só recentemente ganhou repercussão mundial. Tem sido a preferida por A. Wretlind, na Suécia e outros grupos.

Nela, utiliza-se lípides como uma das principais fontes de calorias; a diferença consiste na substituição de parte de glicose por gorduras. Isto significa que podese entregar glicose à $10 \%$, junto com os outros elementos, administrando-se, porém, cerca de 30 a $40 \%$, até $50 \%$, de suas necessidades calóricas globais em forma de emulsão lipídica a $10 \%$, diariamente. Este esquema apresenta uma osmolaridade média, e pode ser infundido por veias superficiais ou periféricas.

c. Nutrição Parenteral Incompleta - onde se utiliza aminoácidos isoladamente, como método de economizar proteínas do paciente, durante fases curtas de carência nutricional. Este esquema contribui para que o paciente que não se alimenta, perca menos proteínas, e é inadequado para nutrição além de sete dias (DICKSTEIN, et alii, 1979).

\section{Contra-indicações e complicações}

Atualmente, existe apenas um grupo de situações que contra-indicam a nutrição parenteral. São aquelas em que há instabilidade circulatória, prejudicando o transporte dos elementos infundidos até a periferia dos tecidos; nesta situação, o acúmulo excessivo desses elementos na circulação, pode gerar os mais variados problemas metabólicos.

Temos, basicamente, três modalidades de complicações:

a) aquelas inerentes à cateterização do sistema venoso central - pneumotórax ou hemotórax, são as mais temidas.

b) aquelas relacionadas com a hiperalimentação parenteral propriamente dita - prende-se mais a uma terapêutica mal formulada, sendo a mais freqüente a hiperglicemia.

c) aquelas ligadas ao tempo de permanência do cateter são as mais freqüentes e mais graves, causando a infecção. Essa complicação excepcionalmente depende do paciente em si (presença de estado septicêmico ou bacterêmico pré-existente); na maioria dos casos, os germes se originam da contaminação da solução ou da linha de infusão, durante o seu manuseio.

\section{Nutrição Parenteral Domiciliar (N.P.D)}

Como ampliação da nutrição parenteral, tradicionalmente hospitalar a nível de Brasil, temos a sua utilização domiciliar ou ambulatorial, a exemplos de casos descritos por outros países, como o Canadá, EUA e França (SOLASSOL \& JOYEUX, 1978).

Essa perspectiva atende a uma clientela que até então não tinha condições de se manter afastada do ambiente hospitalar, em função de sua patologia de base, tais como o eram, também, os pacientes renais em diálise peritonial e hemodiálise.

\section{Indicações}

Vários pacientes precisam de NPD por um período limitado, como coadjuvante do tratamento, ou como terapia primária para certos casos, tais como:

a. Distúrbios gastro intestinais e doença intestinal crõnica fatal - Doença de Crohn; Síndrome de Gardner; Fístula íleo-jejunal com pancreatite crõnica; Infarto das veias mesentéricas.

b. Oncologia - antes e durante a terapêutica quimioterápica; no decurso da radioterapia. Esses pacientes apresentam caquexia secundária à anorexia ou obstruções altas nos tumores de cabeça, pescoço e esõfago, ou ainda, tumores abdominais; além do que a poliquimioterapia agride o epitélio da mucosa gastro intestinal, com sintomas secundários severos.

$\mathrm{Na}$ radioterapia, além do já exposto, há a imunodepressão, que leva a necessidade de um suporte nutricional adequado, a fim de prevenir a debilidade orgãnica.
c. Uremia crônica.
d. Certos casos de hepatopatias e outras.

\section{Acesso Vascular para NPD}

Para o acesso vascular para nutrição parenteral prolongada, atinge-se uma colateral do tronco venoso escolhido, sob anestesia local, dissecando-a até a confluência com o vaso principal. A este nível, é cateterizada sem penetrar no seu interior. A seguir, fixa-se a cãnula ao vaso, com um fio delicado, e a exteriorização é feita por túnel subcutâneo longo, com adequada fixação à pele. (SOLASSOL \& JOYEUX, 1980)

Em geral,para doenças subdiafragmáticas, escolhese $o$ cateterismo superior e para as supradiafragmáticas, utiliza-se o cateterismo inferior, tendo como material ideal, um cateter de Silastic com ponta de teflon (cateter de Scribner - Broviac).

Observamos, pela literatura, que a assistência a esses pacientes, 0 apoio material, realização de programas de saúde, a estrutura social, muito contribuem para a consecução deste tipo de tratamento.

Podemos deduzir, primeiramente, que os pacientes atendidos têm um bom nível cultural, uma vez que eles mesmos preparam a sua solução no domicílio, donde poder-se-ia dizer que são apenas bem treinados, porém para o preparo e aplicação da nutrição, são utilizados uma série de critérios para se atender a uma correta antissepsia os quais não seriam de possível compreensão, caso não houvesse um nível aceitável de esclarecimento por parte do enfermo.

Em relação ao apoio citado, notamos que para a infusão domiciliar da nutrição parenteral, são utilizados materiais que consideramos muito especializados 
para a nossa realidade, sendo que alguns deles nos são familiares somente através de foto ou de visita aqueles centros. São eles: bomba de infusão portátil acoplada ao sistema e ao paciente, durante a infusão; carro especialmente desenhado, semelhante ao nosso "carrode-emergência", que o paciente mantém em casa e que contém todo o material necessário para o procedimento, desde seringa com dose única de heparina até o frasco para a solução; venda, à nível comercial, de soluções de nutrição previamente preparada e doses únicas de medicamentos (seringas com heparina, vitamina K ou B); auxílio por inatividade, pago pelo governo e outros.

0 paciente, anteriormente a sua alta, participa de um curso que consta basicamente de três fases onde se avalia o seu desempenho no auto-cuidado até que seja considerado apto,retornando periodicamente para reavaliações. Têm, para caso de emergência, uma linha telefõnica à sua disposição, 24 horas por dia ( "Hot Line").

\section{OBJETIVO}

O objetivo geral proposto pelo grupo de trabalho é a criação de um Serviço de Nutrição Parenteral Domiciliar (SNPD) em hospitais de Ensino ou Governamentais.

\section{IMPLANTAC̣ÃO E DESENVOLVIMENTO DO PROGRAMA}

\subsection{Proposta de Criação do SNPD}

Tendo em vista os estudos avançados, experiências relatadas e os resultados comprovadamente satisfatórios da terapêutica com Nutrição Parenteral, inclusive no Brasil, acreditamos na grande possibilidade de implantação do SNPD aqui, adaptada às condições de nossa realidade.

Várias iniciativas já foram realizadas em caráter experimental em alguns hospitais como Santa Casa de Misericórdia de São Paulo (RASSLAN ET ALII, 1983), Beneficência Portuguesa e Hospital de Clínicas da Faculdade de Medicina da Universidade de São Paulo (BRITTO ET ALII, 1979). Contudo, não contam com infra-estrutura necessária e não funcionam continuadamente.

A nossa proposta é a criação de um Serviço de NPD inicialmente por um hospital-escola, com apoio e recursos da entidade ou órgão governamental e que funcione em caráter permanente.

O Serviço necessitará de uma equipe multiprofissional com atendimento a todas as unidades do hospital, onde uma enfermeira atuará com exclusividade e centralizará o funcionamento do Serviço. Deve existir uma sala própria para atendimento e recursos para educação e treinamento do paciente e familiares.

$\mathrm{O}$ controle e tratamento se concentrará numa Fi- cha Multiprofissional (FMP) e uma Ficha de Controle Diário (FCD) individualizadas.

\subsection{Atividades dos elementos da Equipe Multi- Profissional do SNPD}

FARMACÊUTICO/BIOQUÍMICO

- Prepara a prescrição médica para NPD;

- Centraliza os recursos necessários para a manuten. ção da NPD;

- Controla a qualidade e esterilidade das soluções, materiais e equipamentos que compõem o Kit para NPD.

ENFERMEIRA

- Planeja, organiza, orienta, treina, executa e avalia a Assistência de Enfermagem ao paciente com NPD e seus familiares;

- Pré e Pós-consulta de enfermagem nos retornos ambulatoriais;

- Encaminha aos demais profissionais;

- Realiza visita domiciliar para acompanhamento sistemático.

MÉDICO

- Prescreve os componentes da NPD e demais terapêuticas medicamentosas $\mathrm{s} / \mathrm{n}$.;

- Estabelece a via de acesso para o cateter;

- Implanta o cateter;

- Avalia o estado clínico do paciente.

NUTRICIONISTA

- Avalia o estado nutricional do paciente;

- Orienta dieta por VO, caso a NPD seja parcial;

- Elabora cardápios para pacientes e familiares.

ASSISTENTE SOCIAL

- Avalia a condição sócio-econõmica-cultural do paciente;

- Levanta recursos da comunidade e entidades quando necessário;

- Controla e providencia os retornos;

- Realiza visita domiciliar para levantamento de dados.

PSICÓLOGO

- Avalia o estado psicológico do paciente e familiares;

- Planeja, executa e avalia a terapia de apoio quando necessário.

\subsection{Programa de funcionamento do SNPD.}

\section{FASE (PACIENTE INTERNADO)}

a. AVALIAÇÃO DA VIABILIDADE DA NPD, CONSIDE-

RANDO:

- estado geral; condições sócio-econõmica-culturais; motivação do paciente; motivação dos familiares;

b. ESTABELECIMENTO DA VIA E IMPLANTAÇÃO DO CATETER.

c. EXPERIMENTAÇÃO DA NP ATRAVÉS DA VIA ESCOLHIDA.

d. EDUCAÇÃO DO PACIENTE E FAMILIARES.

e. PREPARO DO PACIENTE PARA ALTA HOSPITALAR. 
A alta deve ser gradativa, iniciando-se com 2 licenças nos finais de semana, assim definidas:

- primeira licença: saída sábado à noite, com retorno domingo à noite.

- segunda licença: saída sábado de manhã, com retorno segunda-feira de manhã.

f. ALTA HOSPITALAR, com os seguintes encaminhamentos:

Recebe os materiais, equipamentos e medicamentos necessários;

É orientado quanto à data de retorno (1 semana);

Recebe a Ficha de Controle Diário;

Recebe o telefone do SNPD para qualquer intercorrência e recebe endereço da entidade próxima do domicílio, em caso de emergência.

\section{2 a.FASE (PACIENTE NO DOMICÍLIO, COM RETOR- NO SEMANAL).}

a. PRÉ-CONSULTA DE ENFERMAGEM, onde são tomadas as seguintes medidas:

Avaliação do estado geral quanto aos sinais vitais, peso, glicosuria, hidratação, funções fisiológicas

Verificação da FCD (Ficha de Controle Diário); teter;

Observação do aspecto do local de inserção do ca-

Levantamento de eventuais problemas referidos pelo paciente e familiares;

Coleta de sangue e urina para exames de laboratório de rotina (glicemia, $\mathrm{Na}, \mathrm{K}$, Cloro, $\mathrm{Ca}, \mathrm{Mg}$, $\mathrm{P}$, osmolaridade sérica, creatinina, albumina, urina I, glicosúria, cetonúria e outros);

Encaminhamento aos demais profissionais do SNPD e serviços complementares.

b. PÓS-CONSULTA DE ENFERMAGEM, envolvendo:

Reforço e esclarecimento das orientações recebidas pelos profissionais;

Reforço do programa de educação, com devolução de técnicas, mediante material pertencente ao SNPD;

Agendamento do próximo retorno;

Aplicação dos suplementos da NP (vitaminas, Zn, $\mathrm{Fe}$, etc) conforme necessidade.

\section{FASE (PACIENTES NO DOMICÍUO COM RETOR- NO QUINZENAL)}

Nesta fase são repetidos os procedimentos da fase anterior com um prazo maior, pois o paciente já se encontra em condições de prover seu auto-cuidado.

0 processo pode ser interrompido devido à necessidade de reinternação, retorno à $2^{a}$. fase, óbito, suspensão da NPD e outras;

Deve ser feita visita domiciliar pela enfermeira, nos intervalos intercalando-a dos retornos ambulatoriais, quando avaliará as condições bio-psico-sociais do paciente e o encaminhará caso for necessário.

\section{4. $O$ "KIT"da SNPD}

Para que o paciente possa receber sua nutrição em casa, foi criado um "KIT" que contém o material ne- cessário para tal. Isto porque, no Brasil, ainda é inviável ao paciente ou familiares prepararem a solução em casa. Optou-se então por fornecer ao paciente todos os materiais e medicamentos que ele necessitará, para 7 dias, facilitando seu tratamento e evitando erros que ocasionariam graves transtornos.

O "KIT" é composto por uma caixa de isopor e contém:

7 bolsas plásticas com a solução de alimentação individualizada;

7 seringas com solução de heparina ('dose única");

7 tampas para a sonda (cateter);

7 envelopes com bolas de algodão e álcool iodado; 1 pinça Pean;

7 pacotes com 5 compressas de gaze;

Seringas com medicamentos prescritos (vitaminas e outros).

Vale explicar, ainda, que as bolsas utilizadas são de plástico siliconizado semelhantes às utilizadas em nosso meio para sangue e CAPD. Têm capacidade para 1000 ml e são preparadas conforme a prescrição médica para o doente indicado sendo, portanto, individualizadas. A bolsa já possui um equipo para ser inserido na sonda de alimentação do paciente.

As seringas mencionadas, são descartáveis, embaladas em plástico já com a solução de $1 \mathrm{ml}$ de heparina para $4 \mathrm{ml}$ de solução fisiológica a $0,9 \%$. Esta solução será injetada no cateter após o término da alimentação, afim de que não haja resíduos de sangue e fibrina. Para evitar o refluxo do sangue, a injeção é feita concomitantemente com o pinçamento da sonda com a Pean.

As tampas para fechar o cateter são semelhantes a tampas de equipo, de plástico duro, adaptáveis ao tipo de cateter. Vêm em envelope plástico estéril e é utilizada uma nova cada vez que se fecha o cateter.

A infusão da solução será feita intermitentemente por 10-12 hs preferencialmente à noite, ou a critério do paciente.

Para antissepsia das mãos do paciente, do cateter e da ponta do equipo, são usadas as bolas de algodão umedecidas com solução de álcool iodado a $2 \%$ e feita secagem com gaze estéril antes de conectar-se o equipo da solução ao cateter do paciente.

As vitaminas prescritas com dias alternados, serão aplicadas pelo paciente, conforme prescrição.

Todo o material contido no "Kit" deve ser devolvido ao Serviço de Farmácia do Hospital, após uma semana, afim de que o paciente receba a seqüência para mais sete dias. $\mathrm{Ou}$, nos casos da visita domiciliar, a entrega/recebimento será feita pela enfermeira.

0 "Kit" deve ser mantido em geladeira a $4^{\circ} \mathrm{C}$, ou em local fresco, afim de se evitar alteração do material pela luz e calor.

\section{CONSIDERAC̣ÕES}

Tendo em vista, a realidade brasileira, onde não existe catéteres para alimentação parenteral de boa 
qualidade, salvo se importados e obviamente onerosos, optamos por utilizar, mesmo não sendo ideal, um cateter de borracha radiopaca siliconizada tipo "SILASTIC", que será inserido no paciente seguindo-se a técnica convencional. Em casos possíveis, utilizar-se-á o cateter de SCRIBNER - BROVIAC já mencionado como sendo o mais adequado.

Ao retornar ao serviço para controle, será coletado material para exames de laboratório, conforme o resultado da ficha de controle diário e do seu estado geral. Normalmente, os exames pedidos são:

- Sangue: glicemia, Na, K, Uréia, Creatinina, $\mathrm{Cl}, \mathrm{Ca}$, $\mathrm{Mg}, \mathrm{Fe}$, Osmolaridade Sérica, Albumina, etc.

- Urina: Urina tipo I, Glocosúria, Cetonúria, etc.

Os componentes restantes, vitamina $\mathrm{K}$, ácido fólico e outros, que não puderem ser administrados pelo paciente, serão feitos pela enfermeira na visita domiciliar ou no hospital, quando do retorno deste para controle.

\section{CONCLUSÃO}

Sabemos que para uma gama grande de pacientes, a esperança de uma sobrevida maior e melher surgiu com a N.P. Nos países ricos e industrializados onde 0 nível de educação do povo é bom, onde existe preocupação do governo para com a saúde, a N.P. tem atingido uma parcela significativa dos pacientes.

Todavia, em nosso meio, quer pela situação políticoeconômica, quer pela desatenção dada à área de saúde, a N.P. não chega a ser reconhecida como um procedimento médico indispensável como o são a antibioticoterapia, as pequenas cirurgias, e outros. Atualmente, os raros "heróis" que conseguem manter, através das muitas impossibilidades, a terapia de N.P., o fazem em instituições de ensino, governamentais ou particulares onde o paciente assume as despesas, parcial ou integralmente.

Sabe-se que a Sociedade Brasileira de Nutrição Parenteral, muito tem se empenhado para que órgãos responsáveis pela saúde do País (Ministério da Saúde, INAMPS) encarem realisticamente este procedimento em nosso meio, e mais, facilitem os mecanismos para sua utilização como recurso à vida, sejam eles, fabricação de material nacional, pagamento adequado aos serviços feitos, cobertura pelos convênios médicos, incentivo à pesquisa e treinamento da população.

Pode-se, contudo, afirmar que os especialistas da área prevêem um mínimo de 10 anos, para que esta terapêutica possa ser melhor difundida e utilizada de modo conveniente para as partes envolvidas.

Valendo-se do já explanado, sugerimos a criação de Serviços de Nutrição Parenteral Domiciliar, em primeira etapa nos Centros de Ensino do País, os quais reunam condições para a manutenção destes. A nossa proposta visa incentivar o ser doente, mediante o conhecimento sobre a sua doença e estimulá-lo ao autocuidado, desenvolvendo-lhe um senso de responsabilidade sobre a sua saúde. Isto, a longo prazo, atingiria toda a comunidade que estaria aos poucos mudando de mentalidade para com o binômio prevenção - manutenção de saúde. Outro aspecto importante seria a redução de custos de hospitalização atualmente sabidamente onerosos, com pacientes que não necessitam permanecer internados e que fariam o tratamento em casa. Assim sendo, retornariam ao seu meio social e teriam condições de serem total ou parcialmente atuantes em suas atividades anteriores à esta terapia.

\section{REFERÊNCIAS BIBLIOGRÁFICAS}

1. BRITO, I.A et alii. Nutrição parenteral prolongada domiciliar: relato de 1 caso em paciente pediátrica. Trabalho apresentado no Congresso Brasileiro de Nutrição Parenteral, Curitiba, 1979.

2. DICKSTEIN, J. et alii. Nutrição parenteral, Jornal Brasileiro de Medicina, Rio de Janeiro, Publicações Científicas. 1979.(Separata)

3. DUDRICK, S.J. et alii. New concepts of ambulatory home hyperalimentation., JPEN, 3: 72, 1979.

4. RASSLAN, S. et alii. Nutrição parenteral domiciliar. Revista Paulista de Medicina, São Paulo, 101(6): 222-27, 1983.

5. SOLASSOL, C. \& JOYEUX, H. A nutrição parenteral simplificada. In: FAINTUCH, J. et alii Alimentação parenteral prolongada, São Paulo, Manole, 1980, p.133-41.

6. __ Nutrição Parenteral Ambulatorial. In: FISCHER, J. Nutriçäo parenteral, Rio de Janeiro, 1978. Guanabara Koogan. 